\title{
The use of neuropathology in Alzheimer's disease
}

\section{Mini review}

Alzheimer's disease (AD) has been historically defined by protein accumulation around critical areas of the brain, i.e. hippocampus. ${ }^{1}$ The hippocampus is a relatively small brain structure located within the limbic system. ${ }^{1}$ Functionally, this particular structure has been correlated with cognitive functions such as learning, memory and spatial navigation. ${ }^{2}$ In line with this reasoning and thinking in terms of brain disease development, is not difficult to imagine hippocampal damage associated with pathologies like AD. ${ }^{1,3}$ Here two proteins are the major players circumscribing the pathological development of $A D$ : amyloid beta $(\mathrm{A} \beta)$ and tau proteins. ${ }^{3,4}$ Although the exact or the mayor mechanism of protein deposition remains under extensive study, the reality is that we do not know how or why these proteins elicit the accumulation process. However, we need to acknowledge that several pieces of evidence are clues of the putative mechanism. Evidence in support of the case for $A \beta$ : we know that gene mutation in the amyloid precursor protein generates a 42 amino acid peptide that is susceptible to extracellular aggregation during the course of $\mathrm{AD}$ development. ${ }^{5}$ This peptide is the main component of the extracellular $A \beta$ plaque (Figure 1, black arrows). In addition, we know that the $A \beta$ plaque presents preferentially a spherical shape that can be clearly observed around the limbic area (Figure 1, black arrows). Unfortunately and despite this remarkable finding, the gene mutation corresponding as causal mechanism only accounts for the hereditary form of AD. ${ }^{6}$ In this regard, the second and most predominant form of $\mathrm{AD}$ is sporadic, and for this particular type, the mechanism remains unknown. ${ }^{6} \mathrm{We}$ need to mention that the sporadic form of $\mathrm{AD}$ accounts for more than 95 percent of the cases. ${ }^{6}$

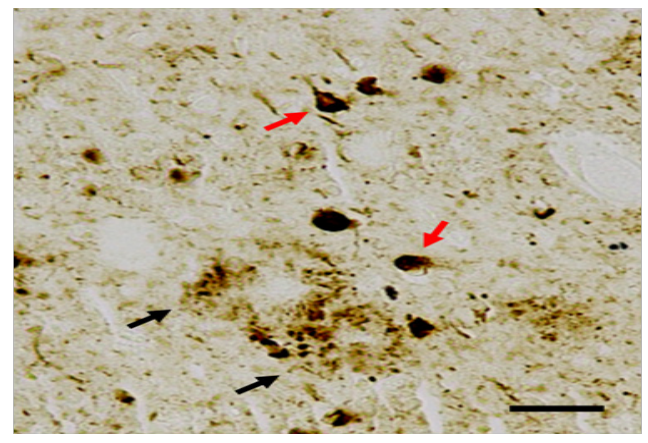

Figure I Phosphorylation of tau protein is a signature of Alzheimer's diseases. Phosphorylation at sites Ser ${ }^{396 / 404}$ labelled by PHF-I are clearly seen in the tau aggregates (red arrows), but importantly, this event is also present in the formation of the $A \beta$ aggregates (black arrows). Scale bar $50 \mu \mathrm{m}$.

Evidence in support of the case for tau protein; of note, remains far more complex and under extensive study. ${ }^{3,6}$ What we know is that tau protein is susceptible of suffering several posttranslational modifications such as conformational shifts, cleavages and several phosphorylation events. ${ }^{1,3}$ The current hypothesis holds that a combination of those events prompts the tau protein to an aggregation state. Thus giving rise to the neurofibrillary tangle (NFT), which normally adopts a flame like shape (Figure 1, red arrows). Important

\author{
Volume 2 Issue 6 - 2016 \\ Siddhartha Mondragón-Rodríguez 1, 2,3 \\ 'CONACyT National Council for Science and Technology, \\ México \\ ${ }^{2}$ UNAM Developmental Neurobiology and Neurophysiology, \\ National Autonomous University of México, México \\ ${ }^{3}$ Department of Psychiatry, McGill University, Canada
}

Correspondence: Siddhartha Mondragón-Rodríguez, Ph.D., Developmental Neurobiology and Neurophysiology, Institute of Neurobiology, National Autonomous University of México. Boulevard Juriquilla 300I, Juriquilla, 76230 Santiago de Querétaro, Qro, México,Tel (442)-238-1057,

Emailsidmonrod@gmail.com

Received: May 16,2016 | Published: August 22, 2016

to note that opposite to the $\mathrm{A} \beta$ plaque which is an extracellular protein deposit, the NFTs are intracellular protein aggregates. ${ }^{1,3}$ In agreement with previous published data, we have observed that the NFTs are mainly comprised of phosphorylase tau protein. ${ }^{3}$

Clearly, both aggregates are related to the $\mathrm{AD}$ pathological development; however the main question is how do the aggregates can affect the hippocampal function? Although the answer is far from simple, one thing is clear; both structures can affect the homeostasis of neuronal function. In one hand the $A \beta$ plaque locates preferentially in the proximity of synaptic terminals with the capacity of modifying the neuronal responses. ${ }^{4,7}$ On the other hand the extensive tau accumulation within the neuron takes all the intracellular space that ultimately leads to neuronal death (Figure 1). Despite this data, we cannot definitively name either of these structures as the cause of the disease. In fact, the chronology of events remains under extensive study. In this vein, we found that phosphorylation of tau protein is an early event that can function as an early stage developmental biomarker in tau pathologies such as Pick disease, Down syndrome and $\mathrm{AD} .{ }^{8,9}$ More recently, by using transgenic mouse models, we have found that abnormally phosphorylase tau protein is actually present before any signs of $A \beta$ peptides (in preparation). This data is extremely important if we take into consideration that $A \beta$ has traditionally been suggested as the effector of tau alterations. ${ }^{10}$ This data opens a new perspective in terms of chronology and pathology effectors. In addition, our data could offer an explanation of the current failure for therapeutic strategies directed against $A \beta$ proteins. ${ }^{10}$ Most likely, we are working with agents directed against the wrong therapeutic target, although this remains to be proven. ${ }^{10}$

Overall, it is clear that both structures, the $A \beta$ plaques and the NFTs, are intimately related to $\mathrm{AD}$ and several associated brain pathologies such as hereditary cerebral hemorrhage with amyloidosis Dutch type, vascular dementia, Pick disease, Down syndrome and Parkinson's disease. ${ }^{3,8,9,11}$ However, only the neuropathological studies will provide a differential diagnosis between brain diseases. 
As a consequence, they remain as the most accurate predictors of neurodegeneration stage.

\section{Financial \& competing interest's disclosure}

The authors have no other relevant affiliations or financial involvement with any organization or entity with a financial interest in or financial conflict with the subject matter or materials discussed in the manuscript.

\section{Acknowledgements}

The authors thank Francis Manno III and Keiry Mariana García Méndez for critical comments and technical support. Siddhartha Mondragón-Rodríguez was awarded a Cátedra support from CONACYT, Mexico.

\section{Conflict of interest}

The author declares no conflict of interest.

\section{References}

1. Basurto-Islas G, Luna-Muñoz J, Guillozet-Bongaarts AL, et al Accumulation of aspartic acid421- and glutamic acid391-cleaved tau in neurofibrillary tangles correlates with progression in Alzheimer disease. $J$ Neuropathol Exp Neurol. 2008;67(5):470-483.

2. Geva S, Cooper JM, Gadian DG, et al. Impairment on a self-ordered working memory task in patients with early-acquired hippocampal atrophy. Dev Cogn Neurosci. 2016;20:12-22.

3. Mondragon-Rodriguez S, Basurto-Islas G, Santa-Maria I, et al. Cleavage and conformational changes of tau protein follow phosphorylation during Alzheimer's disease. Int J Exp Pathol. 2008;89(2):81-90.
4. Kostylev MA, Kaufman AC, Nygaard HB, et al. Prion-Protein-interacting Amyloid-beta Oligomers of High Molecular Weight Are Tightly Correlated with Memory Impairment in Multiple Alzheimer Mouse Models. J Biol Chem. 2015;290(28):17415-17438.

5. Itoh SG, Okumura H. Oligomer Formation of Amyloid-beta (29-42) from Its Monomers Using the Hamiltonian Replica-Permutation Molecular Dynamics 6Simulation. J Phys Chem B. 2016;120(27):6555-6561.

6. Mondragon-Rodriguez S, Basurto-Islas G, Lee HG, et al. Causes versus effects: the increasing complexities of Alzheimer's disease pathogenesis. Expert Rev Neurother. 2010;10(5):683-691.

7. Mondragon-Rodriguez S, Perry G, Zhu X, et al. Phosphorylation of tau protein as the link between oxidative stress, mitochondrial dysfunction, and connectivity failure: implications for Alzheimer's disease. Oxid Med Cell Longev. 2013;2013:940603.

8. Mondragon-Rodriguez S, Perry G, Luna-Muñoz J, et al. Phosphorylation of tau protein at sites Ser (396-404) is one of the earliest events in Alzheimer's disease and Down syndrome. Neuropathol Appl Neurobiol. 2014;40(2):121-135.

9. Mondragon-Rodriguez S, Mena R, Binder LI, et al. Conformational changes and cleavage of tau in Pick bodies parallel the early processing of tau found in Alzheimer pathology. Neuropathol Appl Neurobiol. 2008;34(1):62-75.

10. Mondragon-Rodriguez S, Perry G, Zhu X, et al. Amyloid Beta and tau proteins as therapeutic targets for Alzheimer's disease treatment: rethinking the current strategy. Int J Alzheimers Dis. 2012;2012:630182.

11. Bornebroek M, De Jonghe C, Haan J, et al. Hereditary cerebral hemorrhage with amyloidosis Dutch type (AbetaPP 693): decreased plasma amyloidbeta 42 concentration. Neurobiol Dis. 2003;14(3):619-623. 